\title{
Segregation and Mapping in the Root-Knot Nematode Meloidogyne hapla of Quantitatively Inherited Traits Affecting Parasitism
}

\author{
Varghese P. Thomas and Valerie M. Williamson
}

Department of Plant Pathology, University of California, Davis 95616.

Current address of V. P. Thomas: Bayer CropScience, 1540 Drew Avenue, Davis, CA 95618.

Accepted for publication 11 April 2013.

\section{ABSTRACT}

Thomas, V. P., and Williamson, V. M. 2013. Segregation and mapping in the root-knot nematode Meloidogyne hapla of quantitatively inherited traits affecting parasitism. Phytopathology 103:935-940.

The root-knot nematode Meloidogyne hapla can reproduce on a wide range of crop species but there is variability in host range and pathogenicity both within and between isolates. The inbred strain VW9 causes galling but does not reproduce on Solanum bulbocastanum clone SB22 whereas strain VW8 causes little galling and reproduces poorly on this host. Comparison of reproduction on SB22 of nematode F2 lines generated from hybrids between strains VW8 and VW9 revealed that, whereas over half the lines produced no progeny, some lines reproduced to higher levels than did either parental strain. Using a genetic map previously generated using the same set of F2 lines, three quantitative trait loci (QTLs) were identified and positioned on linkage groups. A combination of two QTL alleles from one parent and one from the other was highly represented in F2 lines that exhibited higher reproduction than either parental strain but was absent from lines that failed to reproduce on SB22. This result suggests that sexual hybridization and assortment of opposing alleles leads to segregation of individuals with improved reproductive ability on a particular host. The genome sequence and integrated genetic and physical linkage map of $M$. hapla provide resources for identification of genes responsible for the identified QTL.
Root-knot nematodes (RKNs) (Meloidogyne spp.) are obligate endoparasites that cause economical damage globally to a wide range of crops (28). The second-stage juveniles (J2s) hatch from eggs in the soil and must find an appropriate host, mechanically penetrate the plant root, and migrate to the vascular tissue to complete their life cycles. These biotropic pathogens then establish complex feeding structures with modified plant cells called giant cells (GCs) that serve as a source of nourishment for the developing worm (35). The cortical tissue surrounding the GCs generally exhibits hyperplasia and hypertrophy, leading to the formation of the characteristic galls or root knots of this genus. Within the gall, the nematode becomes sedentary and develops into a pear-shaped female, depositing its eggs into an egg mass in a gelatinous matrix at the surface of the root.

How the nematode evades host defenses and produces the host cell modifications needed to complete its development is just beginning to be deciphered $(11,12)$. Differential gene expression studies and expressed sequence tag (EST) analyses, much of them focused on genes whose products are secreted from nematode esophageal gland cells, have identified several candidate effector genes. Many of these ESTs have no homology to functionally annotated genes in public databases $(8,13)$, and tools for functional analysis are limited because there is no transformation system. However, the use of RNA interference (RNAi) for gene silencing has met with some success $(17,26)$ and, for several genes, phenotypes have been observed by expressing candidate effector genes in plants $(7,16)$.

Meloidogyne hapla is a major parasite of a wide range of crops, including tomato, potato, carrot, lettuce, alfalfa, and onion (21),

Corresponding author: V. M. Williamson;

E-mail address: vmwilliamson@ucdavis.edu

http://dx.doi.org/10.1094/PHYTO-03-13-0074-R

(C) 2013 The American Phytopathological Society and causes significant agricultural damage in temperate regions worldwide. We have been developing tools to establish $M$. hapla as a model system for identifying traits contributing to parasitism and survival in RKNs by a combined genetic and genomic approach $(23,29)$. This species was chosen because, in contrast to other major RKN pest species (e.g., M. incognita and $M$. javanica), it is a diploid and reproduces by facultative meiotic parthenogenesis and, thus, is amenable to genetic manipulation $(18,30)$. The small $(50 \mathrm{Mb})$ genome of $M$. hapla strain VW9 has been sequenced and annotated $(1,23)$. In addition, this species displays extensive phenotypic variation within and between isolates in host range $(19,20)$ and can overcome host resistance in diverse species, including alfalfa (Medicago sativa) (10), Solanum spp. (15,32), and common bean (Phaseolus vulgaris) (5), thus providing desirable targets for genetic analysis.

We have carried out a genetic cross between two inbred strains of Meloidogyne hapla, VW8 and VW9, and developed a set of 183 F2 lines. Segregating DNA polymorphisms between the F2 lines were used to produce a genetic map $(18,29)$. The current genetic linkage map comprises $>182$ sequence-anchored molecular markers distributed over 16 linkage groups (LGs) corresponding in number to the haploid complement of chromosomes predicted by karyotyping. Because of novel aspects of meiotic recombination in this species, the F2 lines resemble recombinant inbred lines (RILs) and are homozygous for the majority of markers $(18,29)$. These RIL-like lines can be maintained as cultures on tomato plants and used to assess segregation of phenotypic traits. For example, the parental strains differ in the ability to aggregate into clumps in an in vitro assay. Assays on progeny lines revealed that clumping ability segregates as a simple locus and could be mapped to a genetic locus on LG8 (34).

Although quantitative trait locus (QTL) analysis has been used to identify host traits involved in susceptibility and resistance, this strategy has not been utilized much for pathogens. The availability of F2 lines derived from parental strains of M. hapla with phenotypic differences and a genetic map based on molecular 
polymorphisms between these lines presented us with an opportunity to investigate the utility of QTL mapping for identification of loci contributing to parasitism on a particular host. Several factors contribute to the ability of a nematode to parasitize a particular host, including recognition and attraction to the host root, ability to mechanically penetrate the root tip, and ability to establish a feeding site in the host that is capable of providing sufficient nutrition for the nematode to develop into an adult female and to produce viable offspring (31). In this study, we assess the reproductive output of RKN lines on Solanum bulbocastanum, a diploid, self-incompatible wild potato species from Mexico (38). This host has been investigated as a source of resistance to various RKN species, and $S$. bulbocastanum clone SB22 was found to be heterozygous for gene $R_{M c l(b l b)}$, which confers resistance to $M$. chitwoodi and some isolates of $M$. hapla $(3,27,38)$. Here, we demonstrate that M. hapla strains VW8 and VW9 differ in their ability to parasitize SB22 and apply QTL analysis to identify and map genetic loci that contribute to these differences. We present data indicating that combinations of opposing alleles lead to transgressive segregation; that is, progeny lines with phenotypes exceeding those of either parental strain.

\section{MATERIALS AND METHODS}

Plant and nematode material. A vegetative clone of $S$. bulbocastanum, SB22, was obtained from Dr. Charles Brown, United States Department of Agriculture (USDA) Agricultural Research Service at Prosser, WA (3). The derivation of M. hapla parental strains VW8 and VW9 has been previously described (19). The F2 lines used here are a subset of those developed for the genetic map construction $(18,23,29)$. All RKNs used in this work were maintained on tomato 'VFNT' (S. lycopersicum).

Nematode eggs were extracted from plant roots and cleaned by sucrose floatation as previously described (2).

Evaluation of reproduction and galling. For the greenhouse assays, SB22 stem cuttings were treated with Rootone (TechPac, LLC, Lexington KY) following the directions on the label and planted in a 32-ounce Styrofoam cup with drain holes added and filled with sand. After allowing 3 to 4 weeks for the cuttings to develop roots, plants were inoculated with 10,000 eggs of each F2 line. Three replicates of each nematode F2 line were included. Eight weeks after inoculation, plants were uprooted and their roots gently washed free of sand. Root galling severity (RGS) was assessed on a 0 -to- 4 scale, where $0=$ no galling and $1=1$ to 24 , $2=25$ to $49,3=50$ to 74 , and $4=75$ to $100 \%$ galling of roots (22). The roots were then treated with erioglaucine (SigmaAldrich, St. Louis), which stains the egg masses blue, and egg masses were counted (22). Eggs were released from roots using dilute bleach and collected as described by Hussey and Barker (14). The number of eggs was counted in three or more aliquots of the total egg suspension. All data obtained were subjected to $t$ test or analysis of variance (ANOVA) (SAS Institute, Cary, NC).

To assess nematode development, roots were collected at 8 weeks after inoculation, washed, and stained with acid fuchsin (4). Root pieces with galls were pressed between slides and photographed under a dissecting microscope.

Genetic marker nomenclature. Single-nucleotide polymorphism (SNP) markers are annotated with the letter "C" followed by the contig number and position of the polymorphism on the contig (29) (www.hapla.org).

QTL mapping. A genome-wide QTL scan was run on a framework map of 164 markers on 16 LGs spaced at approximately 5 centimorgans $(\mathrm{cM})$ apart. QTL mapping was performed with QTL Cartographer V2.5 (S. Wang, C. J. Basten, and Z. B. Zeng, Department of Statistics, North Carolina State University, Raleigh). Data were analyzed by composite interval mapping assuming multilocus control of the trait (37). A default setting of walkspeed $2 \mathrm{cM}$, model number 6 , five background parameters, window size
$10 \mathrm{cM}$, and automatic selection of the background parameters was used for all analyses. The experiment-wide threshold significance level for each trait was determined by performing 1,000 permutations at $P \leq 0.05$ on the data (6). The graphical representation of the linkage maps and QTL was prepared using MapChart (33). Because of the non-normal distribution, values for each trait were transformed to $\log (x+10)$ for ANOVA and population distribution, where $x=$ mean trait value.

\section{RESULTS}

Comparison of parasitism of $M$. hapla strains VW8 and VW9 on $S$. bulbocastanum SB22. Preliminary experiments indicated that M. hapla strains VW8 and VW9 reproduced poorly, if at all, on S. bulbocastanum SB22. Examination of SB22 roots revealed that VW9 produced distinct galls at 8 weeks after infection whereas VW8 did not (Fig. 1A and B). However, some egg masses were noted on VW8-infected roots but none were seen with VW9. Examination of roots stained with acid fuchsin revealed that VW8 individuals had developed into egg-laying females whereas VW9 nematodes were arrested in development (Fig. 1C and D).

Reproductive ability on SB22 differs among F2 lines. Ninetytwo F2 lines from the VW8 $\times$ VW9 cross, for which molecular marker data were available and which were used to generate a genetic map (29), were assessed for ability to reproduce on SB22. We used two measurements to assess reproduction: total egg masses/root system and total eggs/root system. Although the number of egg masses per root system addresses the ability of the $\mathrm{J} 2$ to initiate successful feeding sites, the measure of total eggs per root system also takes into account the fecundity of the females. Over half the lines showed no reproduction and, for those that did reproduce, both measurements showed statistically significant differences among the lines $(P<0.01)$ (Fig. $2 \mathrm{~A}$ and $B)$. For 11 lines, reproduction was higher than for either parental strain (Table 1). These lines also produced the most eggs but the rank order for these traits differed, suggesting that fecundity was also a factor; and, in fact, the brood size (eggs per egg mass) does vary (Table 1). Root galling severity produced by F2 lines showed continuous variation, and more lines produced galling than were able to reproduce on SB22 (Fig. 2C).

Identification and genetic localization of QTL contributing to parasitism. The distribution pattern and transgressive phenotypes for reproductive ability on SB22 suggested that these phenotypes were governed by multiple genetic differences between parental strains. Therefore, we scanned the genome for QTLs affecting egg mass number per root. Two map intervals, QTL1 and QTL2 on LG7 and LG13, respectively, showed significant logarithm of the odds (LOD) values (Fig. 3A). For QTL1, the maximum LOD score corresponded in position to marker C918.10372, and the F2 lines with highest egg mass numbers were strongly biased toward the VW9 allele (Table 2). For QTL2, marker C357.81930 showed the peak LOD value and the F2 lines with the highest egg mass numbers were biased toward the allele in parent VW8 (Table 2).

When values for total eggs/root were used to scan the genetic map for QTLs, two significant intervals were also identified, one on LG4 and a second on LG7 (Fig. 3B). For the interval on LG4, QTL3, the maximum LOD value co-localized with marker C804.9358, and the VW9 allele was favored. The trait on LG7 localized to the same position as QTL1. To assess the total impact of these three loci on reproduction, we compared the number of lines with each allele combination for three categories of lines (those with $<2,2$ to 10 , or $>12$ egg masses per root) (Table 3 ). Remarkably, none of the 59 lines producing $<2$ egg masses per root carried the VW9 alleles of QTL1 and QTL3 together with the VW8 allele of marker C357.81930. In contrast, 7 of 11 lines that produced $>12$ egg masses had this allele combination. 
A scan for QTLs contributing to galling severity identified one significant QTL with peak LOD at the same position as QTL1 (not shown).

\section{DISCUSSION}

Although M. hapla strains VW8 and VW9 both reproduce well on $S$. lycopersicum, S. bulbocastanum clone SB22 was not a good host for either strain. However, the two strains appeared to be compromised in different aspects of their ability to utilize this host. Even though VW9 can infect and cause galling on SB22, this strain was unable to complete development. This could indicate that VW9 J2 lack factors required for development or maintenance of the feeding site. Alternatively or in addition, this strain may produce a molecule recognized by the host's basal defenses or a host resistance gene, possibly $R_{M c l(b l b)}(27,38)$. In contrast, strain VW8, although the success rate was low, was able to develop and reproduce even though it caused little galling.

The availability of F2 lines provided us with a unique opportunity to begin to dissect and localize the genes contributing to various aspects of parasitism. Although over half of the F2 lines did not produce any egg masses on SB22, our attempts to map
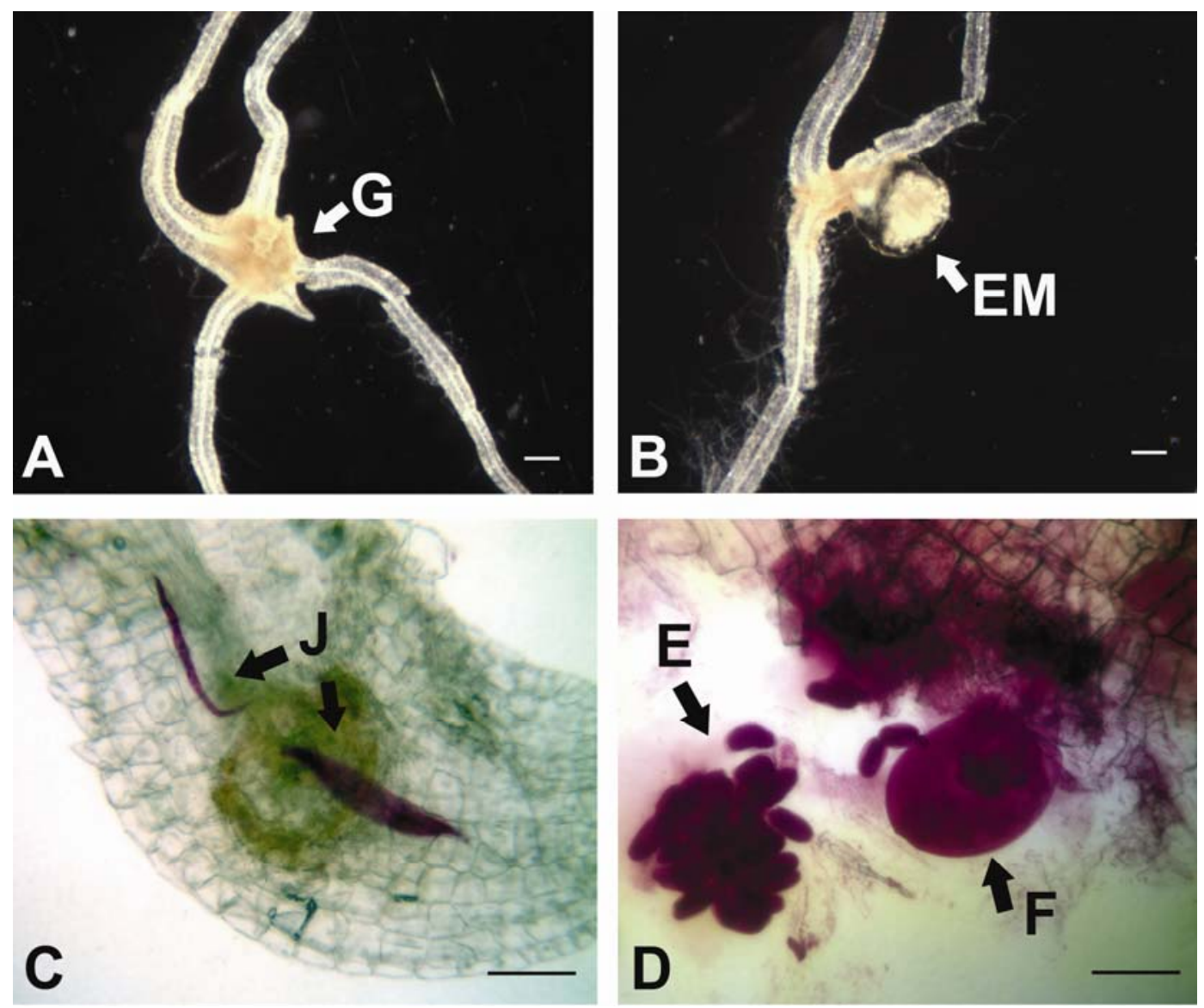

Fig. 1. Comparison of galling and development of Meloidogyne hapla strains VW8 and VW9 on Solanum bulbocastanum SB22. At 8 weeks after infection, A, galls (G) without egg masses were seen on roots infected with VW9 whereas B, egg masses (EMs) without associated galls are seen on roots infected with VW8 C, Staining roots with acid fucshin reveals nematodes arrested in a juvenile (J) stage of development for VW9. D, In contrast, fully developed, bulbous females (F) and eggs (E) can be seen at feeding sites in VW8-infected roots. Size bar represents $0.2 \mathrm{~mm}$.

TABLE 1. F2 lines with highest number of egg masses (EMs) ${ }^{\mathrm{a}}$

\begin{tabular}{|c|c|c|c|c|c|}
\hline \multirow[b]{2}{*}{ RKN strain or line name } & \multicolumn{2}{|c|}{ EMs per root } & \multicolumn{2}{|c|}{ Eggs per root } & \multirow[b]{2}{*}{ Eggs per EM } \\
\hline & Number & Rank & Number & Rank & \\
\hline VW8 & 11.9 & $\ldots$ & 3,918 & $\ldots$ & 329 \\
\hline VW9 & 0 & $\ldots$ & 67 & $\ldots$ & $\ldots$ \\
\hline B35 & 70 & 1 & 46,000 & 1 & 657 \\
\hline A41 & 66.7 & 2 & 3,600 & 9 & 53 \\
\hline B53 & 43 & 3 & 16,260 & 4 & 387 \\
\hline C52 & 42 & 4 & 18,025 & 2 & 429 \\
\hline C13 & 39.5 & 5 & 12,800 & 5 & 324 \\
\hline B57 & 30 & 6 & 3,800 & 8 & 127 \\
\hline A 32 & 18 & 7 & 5,400 & 6 & 300 \\
\hline $\mathrm{C} 54$ & 16.5 & 8 & 18,020 & 3 & 1,092 \\
\hline C18 & 13 & 9 & 3,277 & 10 & 252 \\
\hline $\mathrm{C} 17$ & 12.7 & 10 & 3,198 & 11 & 252 \\
\hline C60 & 12.2 & 11 & 3,823 & 7 & 312 \\
\hline C51 & 10 & 12 & 1,200 & 22 & 120 \\
\hline
\end{tabular}

a VW8 and VW9 are parental strains. The remaining names are F2 lines developed from the parental cross. 


\begin{tabular}{|c|c|c|c|c|}
\hline \multirow[b]{2}{*}{ Egg mass number } & \multirow[b]{2}{*}{ Number of lines } & \multicolumn{3}{|c|}{ Fraction $(\%)$ of lines with VW8 allele at indicated SNP } \\
\hline & & C918.13072 (QTL1) & C357.81930 (QTL2) & C804.9358 (QTL3) \\
\hline$<2$ & 59 & $35 / 59(59)$ & $19 / 59(32)$ & $33 / 59(56)$ \\
\hline $2-10$ & 22 & $8 / 22(36)$ & $13 / 22(59)$ & $5 / 22(23)$ \\
\hline $12-70$ & 11 & 2/11 (18) & $9 / 11(82)$ & 2/11 (18) \\
\hline All lines & 92 & 45/92 (49) & $42 / 92(46)$ & $40 / 92(43)$ \\
\hline
\end{tabular}
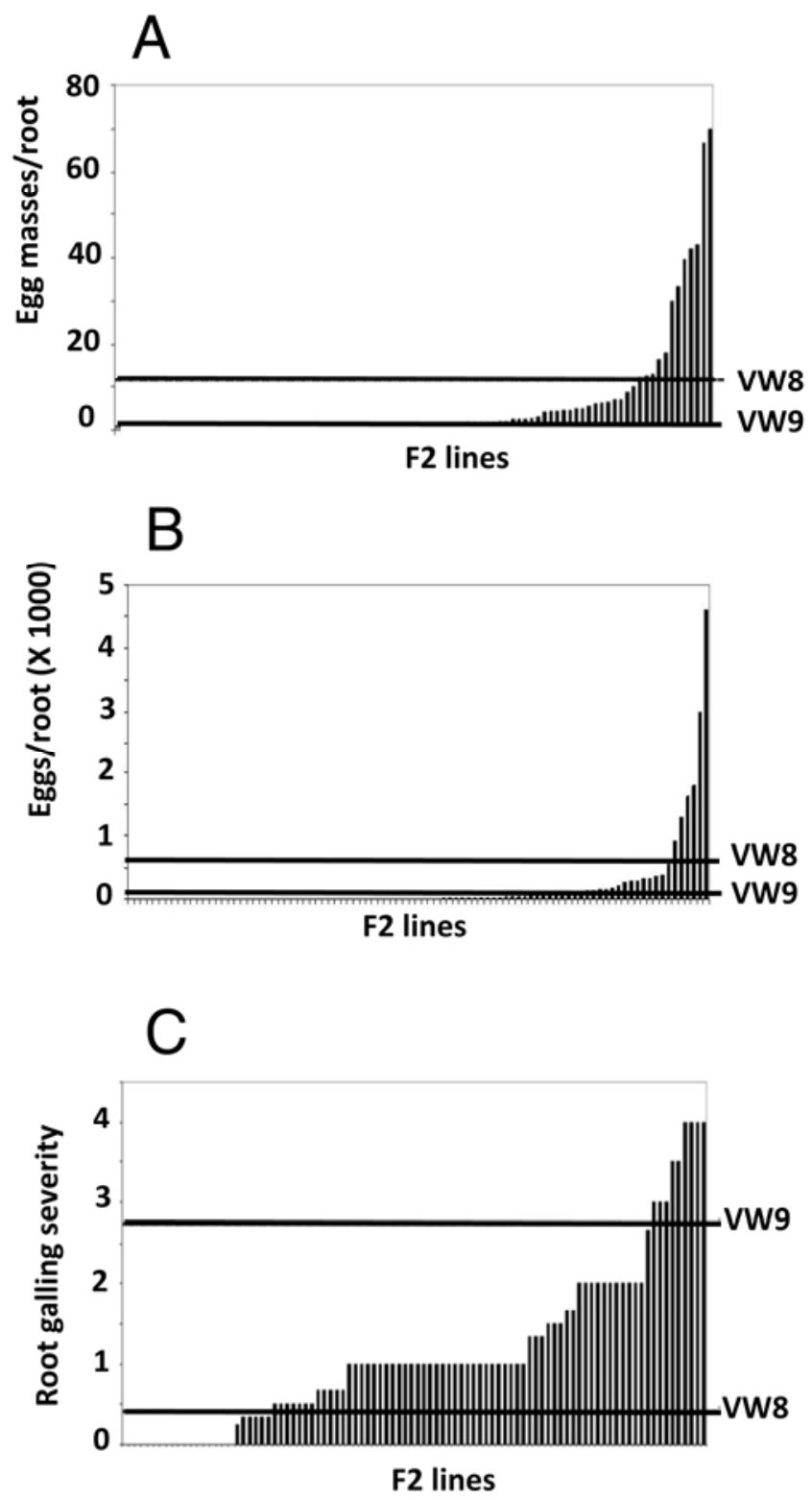

Fig. 2. Distribution of pathogenicity phenotypes of Meloidogyne hapla F2 lines on SB22. The mean number of $\mathbf{A}$, egg masses or $\mathbf{B}$, eggs per root system produced on SB22 infected in each of $92 \mathrm{~F} 2$ lines is shown in ascending order. C, Distribution of root galling severity phenotypes of F2 lines. Horizontal dashed lines in each panel indicate the mean value obtained for each parental strain.

this trait as a single gene locus were not successful, and the continuous distribution of values for the lines that could reproduce suggested that multiple loci contributed to the phenotype. A genome-wide scan identified three QTLs that showed significant LOD values for one or both measures of reproduction. Interestingly, the alleles of QTL1 and QTL3 from VW9, the parental line that could not reproduce on SB22, were favored in progeny lines with the highest reproduction (Table 2). This suggested that
TABLE 3. Distribution of egg mass numbers for each combination of alleles

\begin{tabular}{|c|c|c|c|c|c|}
\hline \multicolumn{3}{|c|}{ Contig allele combination ${ }^{\mathrm{a}}$} & \multicolumn{3}{|c|}{ Number of lines } \\
\hline QTL1 & QTL2 & QTL3 & $<2$ & $2-10$ & $12-70$ \\
\hline B & A & B & 0 & 6 & 7 \\
\hline A & A & A & 5 & 1 & 0 \\
\hline B & A & A & 6 & 2 & 1 \\
\hline B & B & B & 6 & 5 & 0 \\
\hline A & A & B & 8 & 4 & 1 \\
\hline A & B & A & 10 & 1 & 0 \\
\hline A & B & B & 12 & 2 & 1 \\
\hline B & B & A & 12 & 1 & 1 \\
\hline$\ldots$ & $\ldots$ & $\ldots$ & $59^{\mathrm{b}}$ & $22^{\mathrm{b}}$ & $11^{\mathrm{b}}$ \\
\hline
\end{tabular}

${ }^{\mathrm{a}} \mathrm{QTL}=$ quantitative trait loci, $\mathrm{A}$ indicates allele from $\mathrm{VW} 8$, and $\mathrm{B}$ indicates allele from VW9.

b Total lines in each category.

alleles from VW9, even though this parent was unable to reproduce on SB22, contributed to the ability to establish and maintain a successful feeding site. For QTL2, in contrast, the allele from VW8 was highly represented in lines with high egg mass numbers. A possible explanation is that the VW9 allele encodes a nematode effector whose presence is recognized by a host resistance gene (e.g., $R_{M c l(b l b)}$ or other) and triggers an effective defense response, resulting in reduced success of F2 lines with this allele.

Several F2 lines showed transgressive phenotypes (that is, higher reproduction levels than either parent) by both criteria measured. Transgressive segregation has been documented for many organisms and is suggested to contribute to rapid adaptation of a population to new situations (24). Transgressive phenotypes can be caused by combinations of opposing alleles of QTLs in the progeny of a hybrid individual. Consistent with this, the majority of F2 lines with higher egg mass numbers than either parent have an allele combination of QTL1 and QTL3 from parent VW9 together with the VW8 allele of QTL2 whereas none of the 59 lines that produced no egg masses have this combination. The relatively large chromosome complement and high recombination rate of M. hapla should result in rapid reassortment of alleles in progeny of a hybrid and likely contribute to the within-population variation found in this nematode species and the difficulty in obtaining useful host resistance.

Many F2 lines that did not produce any eggs were able to produce galling, indicating that production of galling is not sufficient for a successful feeding site. Other studies have found that root galling and susceptibility or resistance are not always coupled in RKN-host plant interactions $(9,25,36)$. The criteria for measuring root-galling severity are somewhat subjective because the value used is based on assessing both size of galls and distribution of galls on the root. Nevertheless, the QTL2 allele that contributed to reproductive success was also identified as contributed to galling severity, indicating that galling may contribute to or be facilitated by a successful interaction.

Future identification of the genes responsible for the identified QTL will require fine mapping to identify candidates as well as functional assays or additional genetic analysis. The integrated genetic and physical linkage map of $M$. hapla is the most comprehensive for any parasitic nematode and provides an invaluable resource. The genome-wide recombination rate of $M$. hapla has 

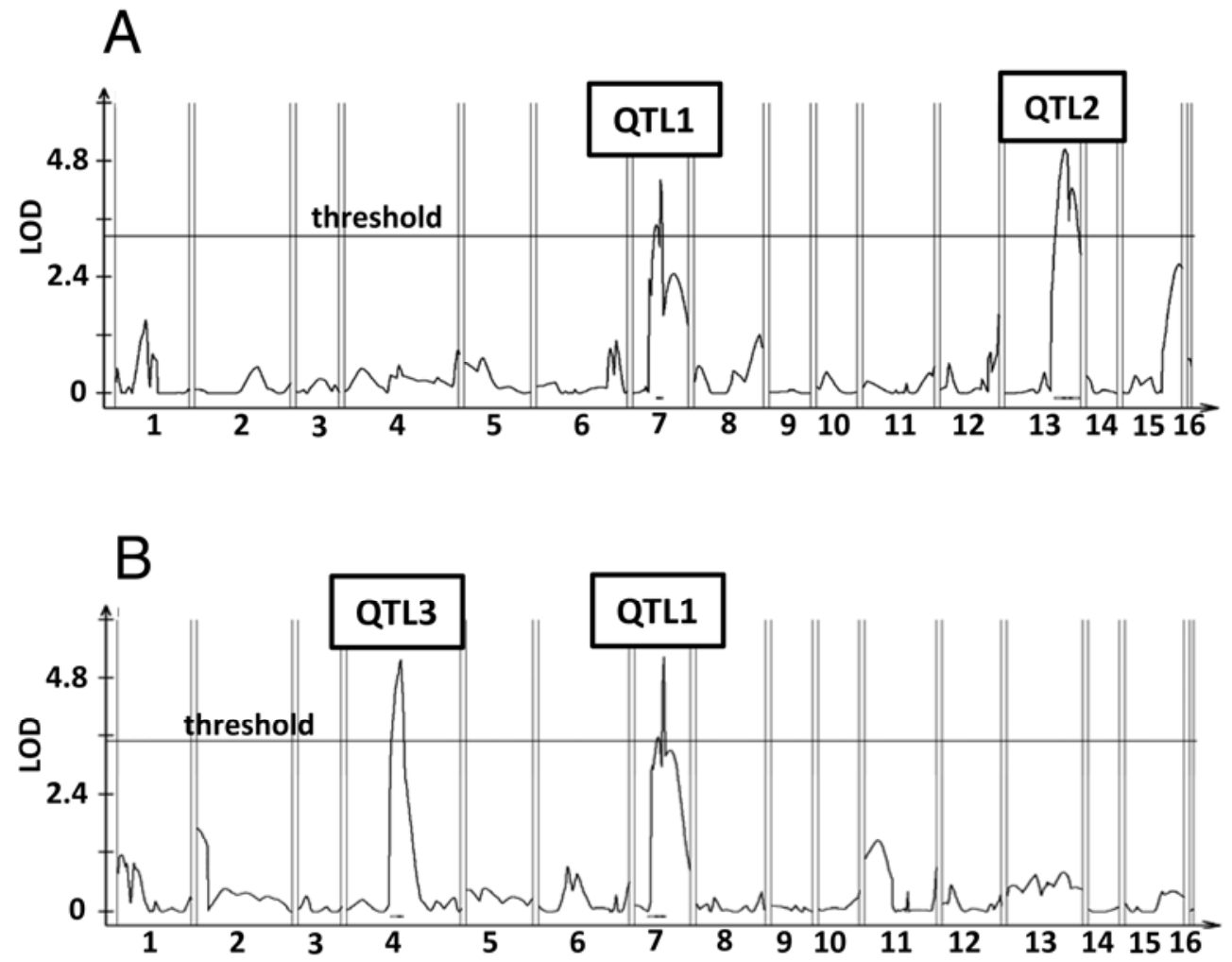

Fig. 3. Genome-wide scan for quantitative trait loci (QTLs) influencing reproduction of Meloidogyne hapla on Solanum bulbocastanum SB22. Markers spanning the 16 linkage groups (LGs) were scanned to locate QTLs contributing to A, egg mass number or B, total eggs among F2 lines. Numbers on the x-axis correspond to the 16 LGs and the Y-axis to the logarithm of the odds (LOD) score. The horizontal line indicates threshold for $95 \%$ significance. Lower panel for each profile indicates allele association with positive values for VW8 allele and negative for VW9 allele.

been estimated to be $135 \mathrm{cM} / \mathrm{Mb}$, the highest known for any metazoan to date (29), and this will facilitate fine mapping. In the case of QTL1, three SNPs have been mapped on Contig918, and analysis of segregation of alleles supports the location of the responsible gene on this 14-kb contig. However, for the remaining QTLs, closely mapped SNPs flanking the gene are not yet available and gaps in the genome sequence prevent us, thus far, from obtaining a physical contig that spans the gene of interest. Mapping additional SNP markers targeted to the QTL region should be feasible because sequence comparison of the parental strains has identified over 20,000 sequence polymorphisms between them (29), and efforts to fill gaps in the genome sequence are in progress. This powerful genetic/genomic strategy should provide new insights into how RKNs manipulate their hosts for successful parasitism.

\section{ACKNOWLEDGMENTS}

This work was supported by funds from National Science Foundation (award numbers IOS-0744857 and IOS-1025840). We thank C. Brown, USDA, Prosser, WA, for providing us with a vegetative clone of SB22; M.-J. Truco for advice on QTL analysis; and D. Bird and G. Bruening for helpful comments on the manuscript.

\section{LITERATURE CITED}

1. Bird, D. M., Williamson, V. M., Abad, P. M., McCarter, J., Danchin, E. G. J., Castegnone-Sereno, P., and Opperman, C. 2009. The genomes of rootknot nematodes. Annu. Rev. Phytopathol. 47:333-351.

2. Branch C., Hwang, C. F., Navarre, D. A., and Williamson, V. M. 2004. Salicylic acid is part of the Mi-1-mediated defense response to root-knot nematode in tomato. Mol. Plant-Microbe Interact.17:351-356.

3. Brown, C. R., Yang, C. P., Mojtahedi, H., and Santo, G. S. 1996. RFLP analysis of resistance to Columbia root-knot nematode derived from Solanum bulbocastanum in a $\mathrm{BC}_{2}$ population. Theor. Appl. Genet. 92:572576.

4. Byrd, D. W., Jr., Kirkpatrick, T., and Barker, K. R. 1983. An improved technique for clearing and staining tissues for detection of nematodes. J. Nematol. 15:142-143.

5. Chen, P., and Roberts, P. A. 2003. Virulence in Meloidogyne hapla differentiated by resistance in common bean (Phaseolus vulgaris). Nematology 5:39-47.

6. Churchill, G. A., and Doerge, R. W. 1994. Empirical threshold values for quantitative trait mapping. Genetics 138:963-971.

7. Doyle, E. A., and Lambert, K. N. 2002. Cloning and characterization of an esophageal-gland-specific pectate lyase from the root-knot nematode Meloidogyne javanica. Mol. Plant-Microbe Interact. 15:549-556.

8. Dubreuil, G., Magliano, M., Deleury, E., Abad, P., and Rosso, M. N. 2007. Transcriptome analysis of root-knot nematode function induced in the early stages of parasitism. New Phytol. 176:426-436.

9. Garcia, G. M., Stalker, H. T., Shroeder, E., and Kochert, G. 1996. Identification of RAPD, SCAR, and AFLP markers tightly linked to nematode resistance genes introgressed from Arachis hypogaea. Genome 39:836845 .

10. Griffin, G. D., and McKenry, M. V. 1989. Susceptibility of Nevada synthetic XX germplasm to a California race of Meloidogyne hapla. J Nematol. 21:292-293.

11. Haegeman, A., Mantellin, S., Jones, J. T., and Gheysen, G. 2012. Functional roles of effectors of plant-parasitic nematode. Gene 492:19-31.

12. Hewezi, T., and Baum, T. J. 2013. Manipulation of plant cells by cyst and root-knot nematode effectors. Mol. Plant-Microbe Interact. 26:9-16.

13. Huang, G, Gao, B., Maier, T., Allen, R., Davis, E. L., Baum, T. J., and Hussey, R. S. 2003. A profile of putative parasitism genes expressed in the esophageal gland cells of the root-knot nematode Meloidogyne incognita. Mol. Plant-Microbe Interact. 16:376-381.

14. Hussey, R. S., and Barker, K. R. 1973. A comparison of methods of collecting inocula of Meloidogyne spp., including a new technique. Plant Dis. Rep. 57:1025-1028.

15. Janssen, G., van Norel, A., Verkerk-Bakker, B., and Janssen. R. 1997. Intra- and interspecific variation of root-knot nematodes, Meloidogyne spp. with regard to resistance in wild tuber-bearing Solanum species. Fundam. Appl. Nematol. 20:449-457.

16. Jaouannet, M., Magliano, M., Arguel, M. J., Gourgues, M., Evangelisti, E., Abad, P., and Rosso, M. N. 2013. The root-knot nematode calreticulin Mi-CRT is a key effector in plant defense suppression. Mol. PlantMicrobe Interact. 26:97-105.

17. Lilley, C. J., Bakhetia, M., Charlton, W. L., and Urwin, P. E. 2007. Recent progress in the development of RNA interference for plant parasitic 
nematodes. Mol. Plant Pathol. 8:701-711.

18. Liu, Q. L., Thomas, V. P., and Williamson, V. M. 2007. Meiotic parthenogenesis in a root-knot nematode results in rapid genomic homozygosity. Genetics 176:1483-1490.

19. Liu, Q. L., and Williamson, V. M. 2006. Host-specific pathogenicity and genome differences between inbred strains of Meloidogyne hapla. J. Nematol. 38:158-164.

20. Melakeberhan, H., Douches, D., and Wang, W. 2012. Interactions of selected potato cultivars and populations of Meloidogyne hapla adapted to the Midwest U.S. soils. Crop Sci. 52:1-6.

21. Mitkowski, N. A., Van der Beek, J. G., and Abawi, G. S. 2002. Characterization of root-knot nematode populations associated with vegetables in New York State. Plant Dis. 86:840-847.

22. Omwega, C. O., Thomason, I. J., and Roberts, P. A. 1988. A nondestructive technique for screening bean germplasm for resistance to Meloidogyne incognita. Plant Dis. 72:970-972.

23. Opperman, C. H., Bird, D. M., Williamson, V. M., Rokhsar, D. S., Burke, M., Cohn, J., Cromer, J., Diener, S., Gajan, J., Graham, S., Houfek, T. D., Liu, Q., Mitros, T., Schaff, J., Schaffer, R., Scholl, E., Sosinski, B. R., Thomas, V. P., and Windham, E. 2008. Sequence and genetic map of Meloidogyne hapla: a compact nematode genome for plant parasitism. Proc. Natl. Acad. Sci. USA 105:14802-14807.

24. Rieseberg, L. H., Archer, M. A., and Wayne, R. K. 1999. Transgressive segregation, adaptation and speciation. Heredity 83:363-372.

25. Roberts, P. A., Matthews, W. C., Ehlers, J. D., and Helms, D. 2008. Genetic determinants of differential resistance to root-knot nematode reproduction and galling in lima bean. Crop Sci. 48:553-561.

26. Rosso, M. N., Jones, J. T., and Abad, P. 2009. RNAi and functional genomics in plant parasitic nematodes. Annu. Rev. Phytopathol. 47:207232.

27. Rouppe van der Voort, J. N. A. M., Janssen, G. J. W., Overmars, H., van Zandwoort, P. M., van Norel, A., Scholten, O. E., Janssen, R., and Bakker,
J. 1999. Development of a PCR-based selection assay for root-knot nematode resistance $(R m c 1)$ by a comparative analysis of the Solanum bulbocastanum and S. tuberosum genome. Euphytica 106:187-195.

28. Sasser, J. N. 1980. Root-knot nematodes: a global menace to crop production. Plant Dis. 64:36-41.

29. Thomas, V. P., Fudali, S. L., Schaff, J. E., Liu, Q., Scholl, E. H., Opperman, C. H., Bird, D. M., and Williamson, V. M. 2012. A sequenceanchored linkage map of the plant-parasitic nematode Meloidogyne hapla reveals exceptionally high genome-wide recombination. Genes Genomes Genet. 2:815-824.

30. Triantaphyllou, A. C. 1966. Polyploidy and reproductive patterns in the root-knot nematode Meloidogyne hapla. J. Morphol. 118:404-414.

31. Trudgill, D. L. 1991. Resistance to and tolerance of plant parasitic nematodes in plants. Annu. Rev. Phytopathol. 29:167-192.

32. Van der Beek, J. G., Poleij, L. M., Zijlstra, C., Janssen, R., and Janssen, G. J. W., 1998. Variation in virulence within Meloidogyne chitwoodi, M. fallax, and M. hapla on Solanum spp. Phytopathology 88:658-665.

33. Voorrips, R. E. 2002. MapChart: software for the graphical presentation of linkage maps and QTLs. J. Hered. 93:77-78.

34. Wang, C. L., Lower S., Thomas V. P., and Williamson V. M. 2010. Rootknot nematodes exhibit strain-specific clumping behavior that is inherited as a simple genetic trait. Plos One 5:e15148.

35. Williamson, V. M., and Gleason, C. A. 2003. Plant-nematode interactions. Curr. Opin. Plant Biol. 6:327-333.

36. Williamson, V. M., and Roberts, P. A. 2009. Mechanisms and genetics of resistance, Pages 301-325 in: Root-knot Nematodes. R.N. Perry, M. Moens, and J. Starr, eds. CABI Publishing, UK.

37. Zeng, Z. B. 1994. Precision mapping of quantitative trait loci. Genetics 136:1457-1468.

38. Zhang, L. H., Mojtahedi, H., Kuang, H., Baker, B., and Brown, C. R. 2007. Marker-assisted selection of Columbia root-knot nematode resistance introgressed from Solanum bulbocastanum. Crop Sci. 47:2021-2026. 